\title{
Vascular closure during transcatheter aortic valve implantation: Literature review and experience from University Hospital Centre Split
}

\section{DIvica Kristić' (iD)Andrija Matetićc ${ }^{*}$, (D)Frane Runjićc', (iDNikola Crnčevićc', (D)Jakša Zanchi', (D)Matjaž Bunc²}

'University Hospital Centre Split, Split, Croatia ${ }^{2}$ University Medical Centre Ljubljana, Ljubljana, Slovenia
RECEIVED:

July 30, 2021

ACCEPTED:

August 5, 2021

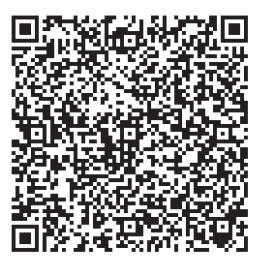

KEYWORDS: vascular closure device, transcatheter aortic valve implantation, vascular access. CITATION: Cardiol Croat. 2021;16(9-10):295-6. | https://doi.org/10.15836/ccar2021.295

*ADDRESS FOR CORRESPONDENCE: Andrija Matetić, Klinički bolnički centar Split, Spinčićeva 1, HR-21000 Split, Croatia. / Phone: +385-98-954-6455 / E-mail: andrija.matetic@gmail.com

ORCID: Ivica Kristić, https://orcid.org/0000-0002-9882-9145 • Andrija Matetić, https://orcid.org/0000-0001-9272-6906 Frane Runjić, https://orcid.org/0000-0001-6639-5971 • Nikola Crnčević, https://orcid.org/0000-0002-1399-3406 Jakša Zanchi, https://orcid.org/0000-0003-2700-2121 • Matjaž Bunc, https://orcid.org/0000-0001-7269-8944

\section{|I||||||||||||||||||||||||||||||||||||||||||||||||||||||||||||||||||||||||||||||||||||||||||||||||||||||||||||||||||||||||||}

Background and Aims: Vascular closure devices (VCDs) have emerged as a routine method for vascular management. Their evolution allowed for further progress and development of interventional cardiology irrespective of the increased frailty of the treated population ${ }^{1}$. This short review aims to present available VCDs and describe the most utilized regimes in everyday practice. In addition, we aimed to present a VCDs protocol at the University Hospital Centre Split.

Materials and Methods: A literature search in the PubMed database was conducted yielding a total of 2,380 research articles in the period of 1975 to 2021. Out of these, 246 articles were review articles. A significant positive trend in the number of published articles was observed across the years (Figure 1). Results: Different types of VCDs exist according to the closure mechanism. Suture-based VCDs include Abbott Perclose ProGlide and Abbott Prostar XL. Plug-based VCDs encompass Terumo Angio-Seal, Terumo FemoSeal, Cordis MynxGrip, Teleflex Manta, Vivasure Medical PerQSeal, and InSeal Medical InSeal $^{2}$. The mechanism of vascular closure by both suture- and plug-based VCDs is presented in Figure 2. Large bore access often requires a combination of the different types of VCDs, and their utilization is literally limitless, depending on the center preferences. Among different options, the most utilized vascular closure method at the University Hospital Centre Split during transcatheter aortic valve replacement (TAVR) combines Abbott Perclose ProGlide and Terumo Angio-Seal allowing for adequate closure and vascular management ${ }^{3}$. After the TAVR finalization, the percutaneous sutures are tightened around an 8 French sheath, followed by the insertion of the Terumo Angio-Seal device according to standards

Conclusions: In conclusion, VCDs represent a modern ubiquitary method for vascular access management, enabling safer and comfortable procedures in a wider patient population. Research on VCDs has substantially increased in recent years. Utilized regimes depend on the access size and center preferences.

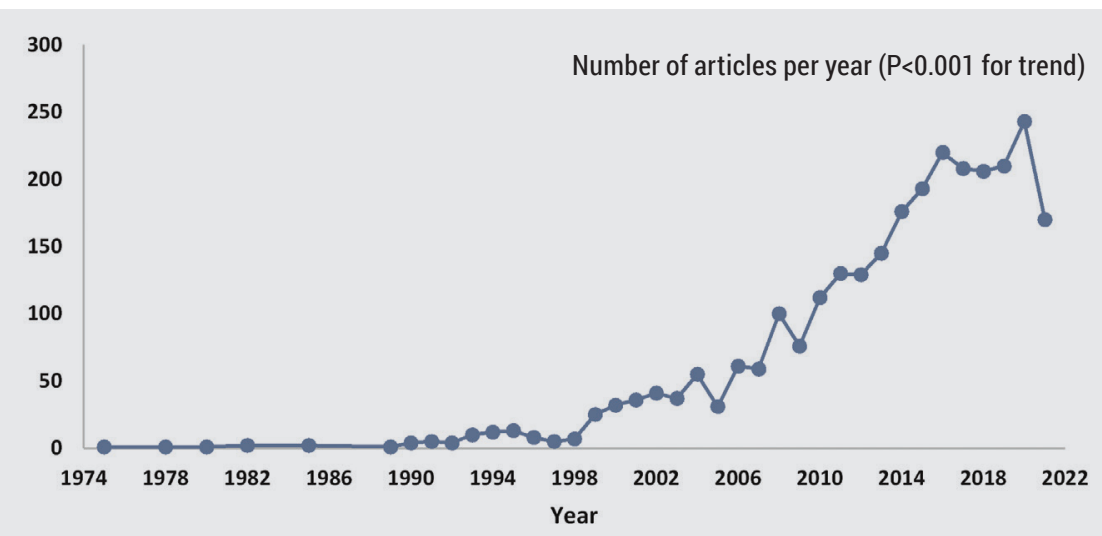

FIGURE 1. Overview of the indexed publications focused on vascular closure devices over the years. 

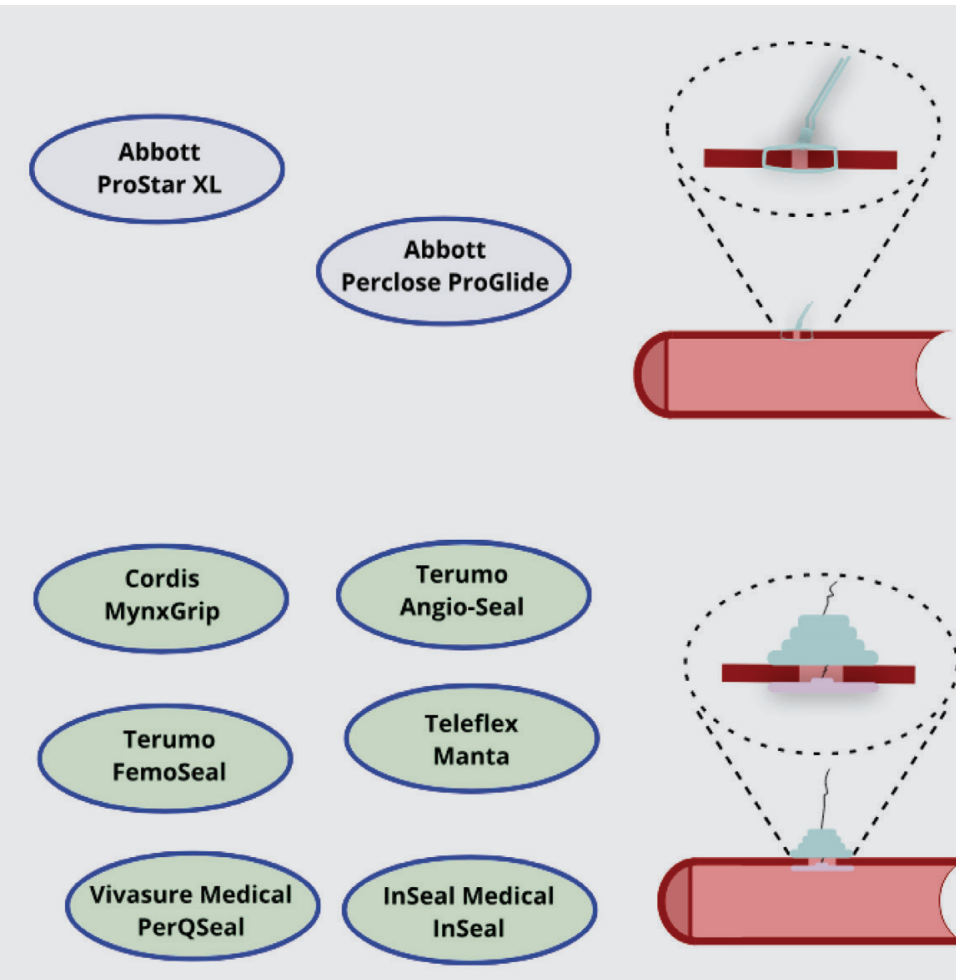

Suture-based mechanism

FIGURE 2. Overview and mechanism of action of different vascular closure devices.

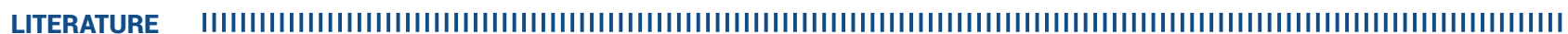

1. Dauerman HL. Rewriting the history of vascular closure devices. EuroIntervention. 2014 Jun;10(2):175-7. https://doi.org/10.4244/EIJV10I2A29

2. Noori VJ, Eldrup-Jørgensen J. A systematic review of vascular closure devices for femoral artery puncture sites. J Vasc Surg. 2018 Sep;68(3):887-899. https://doi.org/10.1016/j.jvs.2018.05.019

3. Ko TY, Kao HL, Liu YJ, Yeh CF, Huang CC, Chen YH, et al. Intentional combination of ProGlide and Angio-Seal for femoral access haemostasis in transcatheter aortic valve replacement. Int J Cardiol. 2019 0ct 15;293:76-79. https://doi.org/10.1016/j.ijcard.2019.05.055 